\title{
The Philosophy of Presence: From Epistemic Failure to Successful Observability
}

\author{
Luciano Floridi \\ Dipartimento di Scienze Filosofiche, Università degli Studi di Bari; Faculty of \\ Philosophy and Sub-Faculty of Computation, Information Ethics Group, Oxford \\ University. \\ Address for correspondence: Wolfson College, OX2 6UD, Oxford, UK; \\ luciano.floridi@philosophy.oxford.ac.uk
}

\begin{abstract}
The paper introduces a new model of telepresence. First, it criticises the standard model of presence as epistemic failure, showing it to be inadequate. It then replaces it with a new model of presence as successful observability. It further provides reasons to distinguish between two types of presence, backward and forward. The new model is then tested against two ethical issues whose nature has been modified by the development of digital information and communication technologies, namely pornography and privacy, and shown to be effective.
\end{abstract}

\section{Keywords}

Cartesianism, environmentalism, epistemic failure, pornography, privacy, successful observability. 


\section{Introduction}

Telepresence is a philosopher's gold mine. It is such a rich concept and experience, a phenomenon so intuitive and yet so difficult to capture in all its nuances and implications, that its potentialities, as a source of new philosophical questions and insights, seem inexhaustible (Ijsselsteijn and Harper [2001] provides a good introduction to presence that prepares the ground for the philosophical debate).

Some of the classic issues in philosophy could easily be reconceptualised as problems concerning (tele)presence (see Goldberg [2000] for a collection of essays concerning several philosophical themes related to telepresence). Examples include action at distance; the semantics of possible worlds understood as the availability and accessibility of spaces different from the actual; the tension between appearance and reality (where is the agent, really?) and the issuing skeptical challenges; testimony as "knowledge at distance" in time as well as in space; the nature of individual identity in different contexts; the mind/body problem; consciousness as awareness of "there-being". Heidegger without the semantics of presence or Dasein would be inconceivable (for a ecological Heideggerian-Gibsonian approach to telepresence see for example Zahorik and Jenison [1998]). And Christian theology has been struggling for centuries with the idea of omnipresence as one of the most significant of God's attributes (the recent debate on divine presence from a telepresence research perspective is reviewed in Biocca [2001], who argues against several conceptual confusions in Sheridan [1999] - who builds on earlier work by Zahorik and Jenison [1998] - and in Mantovani [2001]. On the same debate see also Lauria [2001]).

As the reader will certainly know, the previous list could easily be expanded. Yet this fascinating survey is not the goal of this paper. Instead, in the following pages we shall explore the conceptual foundation of telepresence theory in order to investigate some of the new ethical implications of telepresence (henceforth simply presence, whenever the term causes no confusion). The perspective and methodological tools adopted will be those of the Information Ethics, an environmental approach to Computer Ethics developed by the Information Ethics research Group at the University of Oxford (http://web.comlab.ox.ac.uk/oucl/research/areas/ieg/). 
Here is an overview. The next section provides a critical analysis of the standard approach to the definition of presence as epistemic failure. The identification of some of its shortcomings will lead, in section three, to the elaboration of an alternative conception of presence as successful observability. Once this analysis is laid down, it will become easier to discuss two important ethical issues related to presence. Section four will introduce the environmental approach to computer ethics and a specific application of the new analysis of presence to forms of pornography enabled by digital information and communications technologies (ICTs). Section five moves towards an assessment of the problem of informational privacy, by discussing a possible objection against the interpretation of presence as successful observability. Privacy itself is analysed in section six. The conclusion briefly summarises the results obtained.

\section{The Standard Approach: Presence as Epistemic Failure}

Presence is notoriously a polysemantic concept and a polymorphic phenomenon (Schuemie et al. [2001] and Ijsselsteijn et al. [forthcoming] provide recent surveys of several ways in which presence has been interpreted and analysed, but see also Lombard and Ditton [1997]). However, after almost twenty-five years of research - Minsky [1980] is usually acknowledged as the beginning of presence studies - some convergence on a general conceptual map has begun to emerge (see for example Sacau et al. [2003] and

Ijsselsteijn et al. [forthcoming]). In current studies, presence is often understood as a type of experience of "being there", one loosely involving some technological mediation and often depending on virtual environments. An authoritative and influential source like the International Society for Presence Research (ISPR), for example, endorses the following analysis (italics added):

"[1] Presence (a shortened version of the term "telepresence") is a psychological state or subjective perception in which even though part or all of an individual's current experience is generated by and/or filtered through human-made technology, part or all of the individual's perception fails to accurately acknowledge the role of the technology in the experience. Except in the most extreme cases, the individual can indicate correctly that s/he is using the technology, but at *some level* and to *some degree*, her/his perceptions overlook that knowledge and objects, events, entities, and environments are perceived as if the technology was not involved in the experience. Experience is defined as a person's observation of and/or interaction with objects, entities, 
and/or events in her/his environment; perception, the result of perceiving, is defined as a meaningful interpretation of experience.” (http://www.ispr.info/)

This standard view of presence has been popular at least since the work of Lombard and Ditton [1997]. It consists of three fundamental steps:

i) presence is reduced to a type of perception, e.g. visual perception, for example seeing some geographical shapes and colours;

ii) the type of perception in (i) is then specified, cognitively, as a special kind of experience, namely a psychological, subjective, meaningful interpretation of the experienced; for example, experiencing the above-mentioned colours and shapes as a specific type of environment, e.g. a valley on Mars;

iii) the special kind of experience in (ii) is further qualified, semantically, as a perception of contents that fails, at least partially, momentarily or occasionally, to be a perception of its machine-mediated nature as well; in our example, this means having the impression of being on Mars, failing to realise that it is actually a computer-mediated environment.

Since these three steps are primarily epistemic, one may refer to (i)-(iii) as a model of presence as epistemic failure (the EF model).

The roots of the EF model are (1) philosophically Cartesian and (2) culturally mass-mediatic. An explanation is in order.

1) The philosophically Cartesian nature of EF can be evinced from the priority assigned to the understanding of presence in terms that are exclusively epistemic. In the quotation above from the International Society for Presence Research web site, for example, even the reference to interaction is actually a reference to the perception of interaction. A representative case of a Cartesian approach is Biocca [2001], who defends an approach based on the philosophy of mind and the classic mind-body dualism of Cartesian origins. Note that, although Biocca seems justified in criticizing some metaphysical approaches, this is not a reason to consider Cartesianism the only available alternative.

When Descartes speculates in the Meditations on the possibility of living in a dream or in a Matrix-like reality, somehow artificially generated by a malicious yet omnipotent demon, the stress is precisely on the completely realistic perception of the environment, despite the possibility of an unperceived mediation that makes the perception itself possible yet the environment, and thus our presence within it, entirely 
fictional. Descartes construes the skeptical challenge in terms of a fundamental tension between the actual experience of something - e.g. Descartes being in his room, in front of the fire, looking at his hands - and the possibility of its (i.e., of the perception) unreliability as a source of access to, or presence in front of, the real nature of the experienced something (Descartes suspects he might be dreaming, or might be misled by a malicious demon).

2) The EF model is eminently modern, strictly related as it is to that priority of epistemology over ontology (the conceptual analysis of the ultimate nature of reality) that characterises philosophy after the scientific revolution, from Descartes to Kant. The mass-mediatic character of EF (see especially Lombard and Ditton [1997]) is a reasonable consequence of this Cartesian root. For modernity - known for the primacy it attributes to knowledge and epistemology - makes increasing room for (one may argue that it was bound to lead to) a culture in which the production (fiction) and representation (communication) of realities become socially and psychologically predominant. Simplifying: having placed knowledge at the centre of the stage for some centuries, western thought made almost inevitable the next move, namely the raise of knowledge's products and hence of the infosphere to the key role of primary environment inhabited by the human mind. Correspondingly, the understanding of presence mutates from

a) the mere possibility of an epistemic failure to perceive the difference between what is and what is not real (see Descartes' discussion of the sceptical challenge); to

b) the actual engagement with realities that are known to be artificial or synthetic because demonstrably constructed through the (mass)-mediation of increasingly powerful technologies, which replace Cartesian dreams and demons as the condition of possibility of the experience. In EF, the logical possibility of failure - e.g. one may be dreaming becomes the failure to perceive the technology that may be making one dream.

To summarise, the EF model promotes an understanding of presence as the Cartesian failure to recognize the technologically (mass-)mediated nature of the experiences enjoyed by the epistemic agent. As a consequence, EF allows to catalogue as presence a variety of radically different phenomena otherwise largely unrelated, from oral and textual representations to immersions in VR scenarios, from radio narratives to online games, from television and cinema to tele-robotics. 
It is unclear whether the very wide scope of EF is actually an advantage providing a conceptual reduction of a broad spectrum of phenomena to a single, unifying frame of interpretation - or arguably the sign of some serious misunderstanding. Several reasons may incline one to take the latter view.

$\mathrm{EF}$ embeds an unresolved tension between the subjective, introspective, singleagent understanding of presence - which the model inherits from a Cartesian approach and the social, public, intra-subjective and multi-agent understanding of presence proper of a mass-mediatic approach. Is telepresence a personal and private experience or is it something made possible only by social interaction? Is solipsistic telepresence an oxymoron? Consider just ordinary presence, not telepresence: was Robinson Crusoe present (did he feel present) on the island before meeting Friday? Of course there is no straightforward answer to this type of questions, because, trivially and boringly, it all depends on what one means by "(tele)presence". However, the fact that similar questions are reasonably prompted by the EF model and yet appear so poorly posed is evidence that there might be something wrong with EF itself. EF starts looking like a position that allows misconceived questions to be asked, the sort of questions that make research go amiss.

The previous suspicion paves the way to another, more substantial criticism. EF manages to be, in different ways, both too exclusive and too inclusive, resulting literally eccentric with respect to its correct focus.

On the one hand, by adopting an anthropocentric perspective - typically Cartesian - the model considers beyond its scope any investigation of cases of presence of (not just through, or by means of) artificial agents. And this because, at least at this stage in the evolution of AI, no machine is capable of subjective experience of any sort, let alone one of a Cartesian nature. Yet telepresent robotics is not just about devices remotely controlled by human operators, it is also and significantly about devices that are able to be present remotely by telecontrolling other devices, while keeping human agents entirely out of the loop, as mere external observers. Along the same line, if more hypothetically, it is hard to see how EF can analyse the concept of presence when the agent involved is a cyborg, that is, an agent who may enjoy some technologically-mediated experiences of presence while at the same time perceiving them precisely as mediated. 
On the other hand, the EF model grants full citizenship in the realm of telepresence studies to experiences such as reading a novel or watching a movie, an oddity that causes a loss of specificity and an irrecoverable metaphorization of the concept of "presence at distance”.

This metaphorical way of approaching presence can be related to a further difficulty. EF provides a merely negative understanding (more on this presently) of presence - as failure to perceive the technologically-mediated nature of the experience and this is bound to be unsatisfactory. The approach by negation (per via negativa) means that one attempts to define or conceptually capture a definiendum by saying what the definiendum is not. It may work with dichotomies and Boolean concepts: if one understands what "left" means one may also understand the meaning of "right" negatively, as "not-left". It is a standard method in mathematics, where the the method of false position or regula falsi helps one to estimate the roots of a nonlinear equation $\mathrm{f}(\mathrm{x})=$ 0 by an iterative process of "false" approximations. But "failure to perceive" fails itself to be either a Boolean description or a precise concept that can be further refined by iteration. It is comparable to defining a zebra as not quite a horse but close: it includes far too many things (might it be like a centaur? A mule? A camel?) and, although correct, it begs the question, since we might as well speak of a zebra as not a donkey but almost. That we speak of a zebra in terms of not being a horse - that we conceptualise presence as epistemic failure - only shows that we do already possess some fairly detailed idea of what we wish to define - the zebra in front of us or at least in our memory, the actual experience of being telepresent - but that we surrender to the difficulty of providing a tight conceptual analysis. Instead, we opt for what is in fact a merely generic indication, a “you know what I mean”, a finger-pointing. This brings us to a further problem.

EF allows odd cases of nested telepresence. Consider the Odyssey. A large part of Odysseus’ adventures are recounted by Odysseus himself after having landed to Scheria, the island of the Phaeacians. One of these adventures is the encounter and blinding of Polyphemus in the Cyclops' island. According to EF, the reader, by being in Homer's narrative space, is also in Scheria where, by listening to Odysseus, she is also in the Cyclops' island. Only a semantic space can allow this nesting. But then only a metaphorical sense of "telepresence" may be at work here. For this nesting has nothing to 
do with the ordinary set-theoretic sense in which, by being telepresent in a given space $S_{1}$, say a hotel room, one is also telepresent in the space $S_{2}$ that includes $S_{1}$, say in the hotel where the room is. In the latter case, the co-(tele)presence is a logical necessity. In the former case, it can only be a matter of possible mental experience.

We have reached the last problem. EF cannot clearly define absence. This is not a philosophical gimmick. Any conceptual analysis of telepresence should also be able to discriminate between, and possibly explain, cases of unachieved telepresence, of failure or interruption of telepresence, of faulty or insufficient telepresence. So here lies another clear sign that the EF model is unsatisfactory. Fortunately, it is also the condition of possibility of a better approach.

Consider a counterfactual analysis: had the agent not failed to perceive the technologically-mediated nature of her experience she would not have been telepresent. This is the inevitable logical consequence of EF, but it is also a reductio ad absurdum. For surely the doctor teleoperating on a patient is still present, independently of her perception (or lack thereof) of the technological mediation. Surely the soldier is still telepresent on the mine field through a robot, despite all the possible perception of the artificial nature of the experience. The fact is that epistemic failure is not the right criterion to identify cases of telepresence. The good news is that, precisely by focusing on absence, we can gain a better perspective on presence and hence acquire a vantage point to frame some relevant ethical issues.

\section{A Different Approach: Presence as Successful Observability}

Concentrating on absence has the immediate advantage of clarifying that speaking of presence in a vacuum of references makes little sense. Something is (tele-)present or (tele-)absent only for an observer and only at a given level of abstraction (see Ijsselsteijn [2002] for a similar perspective).

A level of abstraction (LoA) is a specific set of typed variables. Intuitively, it is representable as an interface, although, strictly speaking, this is inadequate, not least because an interface is usually static, whereas a LoA is dynamic. 
Through a LoA the observer accesses the environment, so a LoA could be e.g. the five senses unaided, a microscope, a CCTV or a Geiger counter (on the LoA methodology see Floridi and Sanders [2004]).

Consider a motion detector, for example (Fig.1). In the past, motion detectors caused an alarm whenever a movement was registered within the range of the sensor, including the swinging of a tree branch (object $a$ in Fig. 1). The old $\mathrm{LoA}_{1}$ consisted of a single typed variable, which may be labelled MOVEMENT. Nowadays, when a PIR (passive infrared) motion detector registers some movement, it also monitors the presence of an infrared signal, so the entity detected has to be something that also emits infrared radiation - usually perceived as heat - before the sensor activates the alarm. The new $\mathrm{LoA}_{2}$ consists of two typed variables: MOVEMENT and INFRARED RADIATION. Clearly, a cat (object $b$ in Fig. 1) walking in the garden is present for both LoAs, but for the new $\mathrm{LoA}_{2}$, which is more finely grained, the branch of the tree swinging is absent. Likewise, a stone in the garden (object $c$ in Fig. 1) is absent for both the new and the old LoA, since it satisfies no typed variable of either one.

Space of observation

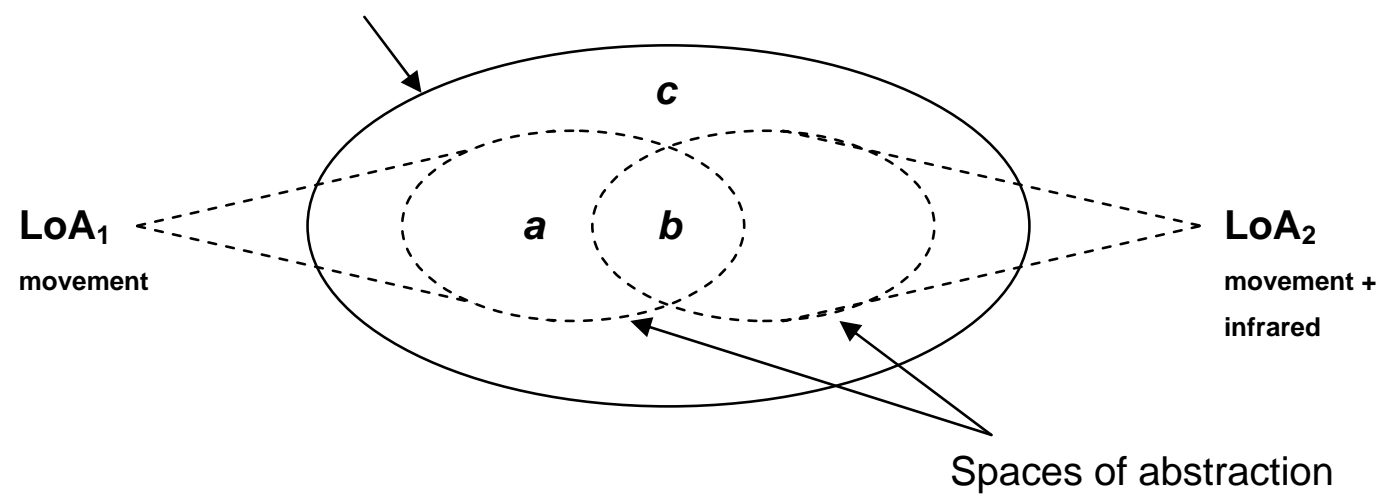

Fig. 1 An example of Levels of Abstraction

What the two sensors detect (the word is used here in a purely engineering sense of extracting data from a signal) is movement, a change in the environment, some form of action (e.g. walking) or interaction (e.g. interrupting the flow of a signal) or a transition 
in the system. More generally, this is one of the two senses in which something is present or absent in a space of observation: as a dynamic source of action/interaction or change.

The other sense is as a static property-bearer. The immobile branch of the tree is absent both for the old-fashioned sensor and for the new PIR sensor. It is still absent for the latter, even if it moves, because it fails to satisfy another typed variable, the infrared one. The cat, on the contrary, is constantly (i.e., non-intermittently) present for an infrared sensor, even if it does not move, because it is a heat-generator.

The method of LoA is an efficient way of making explicit and managing the ontological commitment of a theory. This is crucial. Mantovani and Riva [1999], for example, acknowledge that "[...] the meaning of presence depends on the concept we have of reality (from the ontology which we more or less explicitly adopt) and that different ontological positions generate different definitions of presence, telepresence and virtual presence.” It seems that what is needed is a method of LoA.

The method clarifies that to be present is to be the value of a typed variable of a LoA (to paraphrase Quine). To be absent is, of course, to fail to be any such value. This view is consistent with the general thesis, defended in Mantovani and Riva [1999], that presence is an ontology-dependent concept. The social construction of presence, further supported by Mantovani and Riva [1999] may be interpreted as a specific case of the broader view articulated in this paper.

As we have just seen, depending on the class of typed variables in question, there might be three ways of being present/absent at a given LoA:

1) as source of action/interaction,

2) as a property-bearer,

3) as both (1) and (2).

Without clause (2) one would be unable to define forms of "passive” presence. Thus, a model according to which "presence is tantamount to successfully supported action in the environment." (Zahorik and Jenison [1998]) would fail to acknowledge the fact that $x$ might be present even without any observable (let alone successful) interaction between $x$ and $x$ 's environment. Of course, a solution would be to modify our understanding of “interaction” and "environment”, but this seems rather ad hoc. A more fruitful alternative is to accept that any analysis of presence requires the identification of a space of 
observation and a level of abstraction. Unperceivable subatomic particles are known to be present from their actions and our interactions. The sofa in the room is present because of its perceivable qualities. The flame of a candle in the room is present because of both. Absence may be equally gradual.

If we now extend the previous analysis to telepresence, the easiest thing is to refer to the new model as being based on successful observability (SO), thus:

SO) an $x$ observable at a given LoA in a local space of observation LSO is also telepresent in a remote space of observation RSO if and only if $x$ is also observable in RSO at a given LoA.

Note that LSO and RSO need to be different (LSO $\neq$ RSO), whereas the two LoAs may but do not have to be identical (see Fig. 2).

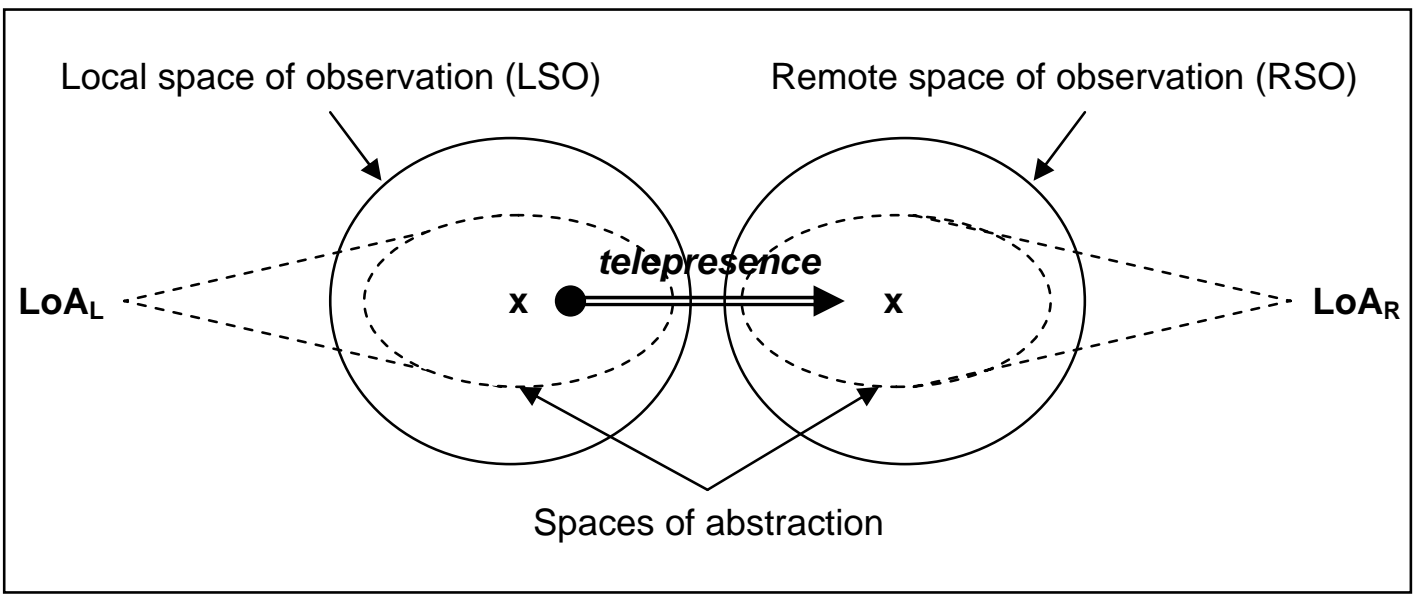

Fig. 2 A model of telepresence

The new model shifts the perspective from an internal and subjective assessment of a peculiar experience - presence as epistemic failure - to an external and objective evaluation - presence as successful observability - which requires an explicit definition of the LoAs adopted in the process of analysis. This has at least four major advantages.

The first and most obvious is that, contrary to the EF model, the SO model provides a clear criterion of discrimination between what does and what does not count as telepresence. It thus regiments very clearly the applicability of the concept, which now excludes intentional experiences, which may be technologically-enabled but are in themselves merely psychological - such as reading, listening to the radio or watching a 
movie - but includes standard cases of presence, such as operating in virtual environments (from immersive virtual realities to text-based virtual worlds such as MUDs, MOOs, IRC, and Chats), remote controlling other artificial agents, being a member of a digital community, playing online. Since there is no presence in a remote space unless the entity in question is observable there at some given LoA, one cannot be telepresent in War and Peace, in Casablanca or on Scheria.

A further advantage of the new model is that all this is good news for mass-media and literature studies as well. For that peculiar experience of "as if I were there”, caused by many forms of communication, will never be properly studied as long as it is catalogued under the wrong heading of telepresence. It requires the development of its own set of conceptual tools. There are, of course, borderline cases, and the new model contributes to explain them. Watching All My Children (http://abc.go.com/daytime/allmychildren/) on TV does not make the audience telepresent, either as a property-bearer or as a source of interaction. However, participating by tele-phone to a tele-vision program does indeed satisfy the criterion laid down by SO, and quite rightly so, for the audience is now capable of some minimal interaction at distance. Indeed, the example shows the need for a deeper understanding of the nature of environments conducive to telepresence. It takes two to interact. Of course, digital ICTs are far more open to the possibility of telepresence than classic mass media, but telepresence is possible even through the latter. The difference lies precisely in the ontological nature of the digital, which not only "augments" the agents' capacities epistemically, but allows the construction of new spaces where the agent can be telepresent interactively. It won't be long before we might be able to experience something like the "wall-to-wall circuit" interactive TV described by Bradbury in Fahrenheit 451:

“She [Helen] didn’t look up from her script again. 'Well, this is a play that comes on the wall-to-wall circuit in ten minutes. They mailed me my part this morning. [...] They write the script with one part missing. It's a new idea. The homemaker, that's me, is the missing part. When it comes time for the missing lines, they all look at me out of the three walls and I say the lines. [...]' 'What's the 
play about?' 'I just told you. There are these people named Bob and Ruth and Helen'.

A third, important advantage of SO is that it enables one to acknowledge a spectrum of ways of being present, from the weak presence of $x$ barely detectable as a mere propertybearer (more on this in section 6) to the strong presence of an agent endowed not only with observable properties, but also with the capacity of acting and interacting (the agent can be the receiver of an action and respond to it accordingly) with the environment, both pragmatically (by doing or changing things) and epistemically (e.g. by observing things locally). Presence is no longer a Boolean concept - as in EF - and SO justifies talks of augmented telepresence, or attempts at making telepresence resilient, and so forth.

The last advantage to be stressed finally leads us to the discussion of some ethical issues, in the next section.

\section{Environmentalism, Teleagents and Telepatients}

Clearly "being there remotely” as a mere property-bearer is far less useful and interesting than being telepresent also as an agent, capable of some successful action and interaction in the remote space. In both cases, however, telepresence, as defined by SO, brings to light the need to analyse ethical problems that, on the one hand, inevitably escape the old EF model (recall the definition of telepresence as a sort of personal experience), and on the other do not seem mere updated versions of the standard problems occurring in everyday life.

The SO model makes explicit that we are confronted by a new ethical context in which teleagents and telepatients interact in technologically-sustained environments. Of course, their actions have moral values and consequences, but our degree of understanding is still low. Needless to say, the slightly sci-fi scenario should not mislead. Million of people already spend an enormous amount of time online, being present in remote spaces in which they both learn and show how to behave. Interestingly, the fact that many of the entities with which human teleagents and telepatients come in contact may be entirely artificial becomes a source of enrichment of our ethical discourse in general. The new scenarios require a sharpening of old conceptual tools and the creation of new ones. For environmentalism acquires a new meaning when one's environment is a 
remote virtual space and the sort of things one interacts with may have a digital, not a biological nature. A specific example will help to illustrate the point.

According to SO and contrary to EF, classic pornography, in the form of texts, pictures or movies, does not generate any form of presence. In this respect, there is no difference between De Sade's Justine and Voltaire's Candide: the reader is still left out of the remote space of observation.

It follows that whatever might be morally significant with old-fashioned pornography it cannot be grounded on an analysis of telepresence. The dialectics of exposure seems much more pertinent, for example. This still holds true when the nature of the media change from analogue to digital: a pornographic DVD, even if it provides some choices and options, fails to represent a case of presence, according to the new model.

Things stand rather differently, however, with new forms of ICT-based "pornography" (the quotation marks are required precisely by the novelty), which implement various degrees of interaction without any form of physical intercourse: dedicated telephone services, chatrooms and other multi-user environments, usually employed for role-playing games, or experimental virtual reality scenarios. Here pornography (which is a semantic concept), promiscuity and prostitution (which are pragmatic concepts) merge. In similar cases, the agent is indeed present remotely, at least in the sense supported by SO, in semantic spaces that also allow some degree of interaction. However, despite the obvious connection with more ordinary form of pornography, one important difference is that the other tele-agents with "whom" the human agent interacts may be entirely synthetic. S1m0ne, the film directed by Andrew Niccol about a digitally created actress "who" becomes a star, offers a great thought experiment. More realistically and less morally, erotic chatterbots nowadays are not science fiction. People have been gallant and tried to date pieces of software. So arguments against pornography based on the crimes, immoralities, degradation, exploitation and health hazards that may affect the people involved - an argument often rehearsed in the context of pornographic videos - may become ineffective. Likewise, any Kantian argument to the effect that no human being should be used as a mere means 
would be inapplicable. Clearly telepresence in informational environments inhabited by agents of unclear nature is forcing us to rethink our well-entrenched, ethical assumptions.

\section{An Objection against Presence as Successful Observability}

Checking the limits of the old EF model, one may be tempted to raise similar objections against the new SO model. True, SO does provide a definition of presence and a criterion of discrimination between presence and absence. But SO might still be eccentric in a very significant way. For one of the most important types of phenomena, commonly interpreted as presence, refers to the availability of tele-perceptual technologies such as radars, satellites, webcams, sonars, CCTVs. It seems that, without being either a property-bearer or a source of interaction in a RSO, a entity, even an artificial one, might still be present in a RSO remotely, e.g. by means of a monitoring appliance. Yet SO fails to accommodate such types of presence, which might be qualified as telepistemic. It follows that SO needs to be revised, if not abandoned.

The previous objection is correct in drawing the inference, but mistaken in suggesting the need for a solution. What needs to be modified is our understanding of teleepistemics itself. For what looks like telepresence is in fact something slightly different, and understanding the difference casts an interesting light on several issues.

Suppose you are in a room. You are just present in that room. Pull down the wall between that room and the next, and you will not say that you are now telepresent in the next room; you are merely present in a larger room. In chess, when a Pawn reaches the opposite side of the board, it can be promoted to any piece except a King. Suppose the Pawn is promoted to a Queen. Suddenly most of the board becomes a local space, distant only one move. Many telepistemic technologies are "tele-promoting" in this sense. The Queen is not telepresent in a remote space, it is the space of the Pawn that has been enlarged. Take a digital camera. Start monitoring what is happening in your room. Again, you are not telepresent in your room, or at least not according to SO (at least because in this case we have LSO = RSO); the burden of proof that you are is on EF's shoulders. Now imagine making the digital camera one inch longer, and then another inch, and so forth. Or just make your camera increasingly powerful. Gradually, the camera allows you to monitor things that are increasingly further away from your local space. At what point 
are you telepresent? At ten metres? Fifty? A hundred? When only a cable connects you and the appliance? Or a radio signal? The answer is never, according to the SO model. Making a remote space epistemically available locally is different from being present in that remote space as an entity. It is like pulling down the wall between two rooms. This is why there is no point in using a portable, baby-monitor unit with a range of several miles: the monitor guarantees to the user only telepistemic access to the remote space but no actual interactive presence at distance. If something happens, it is only the more frustrating to know that nothing can be done in time, given the long distance.

The problem with telepistemics consists in a fallacious confusion between

1) the successful observability of $x$ not only in LSO but also in RSO; and

2) the successful observation by $x$ - which is in LSO - of some $y$ that is present in RSO. The former is a case of ontic telepresence, the latter is a case of epistemic access at distance. The two phenomena are separate and should not be confused. Compare this to the illusion of movement caused by web-browsing: one feels as if one were being uploaded in different spaces, when in reality one is downloading those spaces into one's own.

Should we then abandon any talk of presence in all those cases of technologically mediated telepistemics? Not so fast. Telepistemics may still be a case of presence, it is just that the previous confusion impedes one to see precisely who or what is telepresent where. It is not the observer $x$ in LSO accessing the entity $y$ in RSO that is present in RSO, but exactly the opposite: by being accessed telepistemically, the $y$ in RSO is now also present in the observer's LSO, typically as a mere property-bearer. Using the previous analogy, once the wall is pulled down, you are not remotely present in the other room; it is the chair that was in the other room that is now locally present in your space. In order to fix the distinction it may be useful to speak of forward and backward presence (see Fig. 3). 


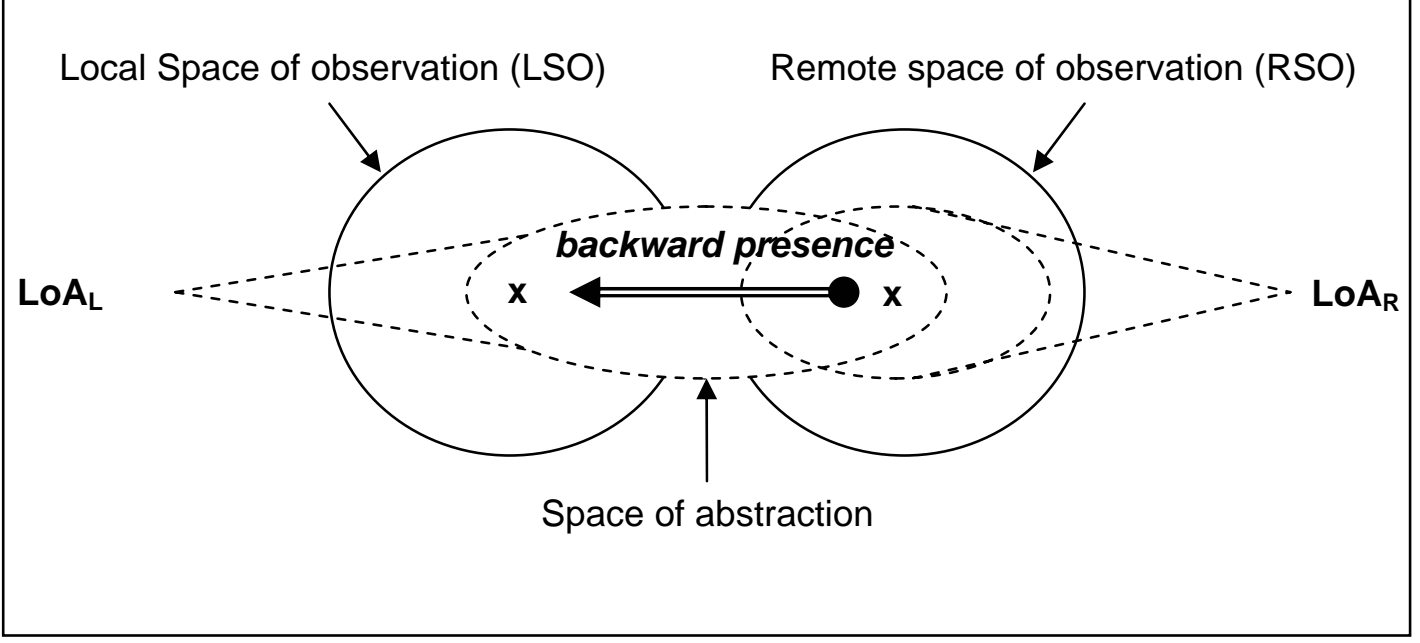

Fig. 3 A model of telepistemics as backward presence

The distinction between forward and backward presence fits SO perfectly well. Recall that something may be telepresent as a mere property-bearer. The map of a portion of the small crater encircling the Mars Exploration Rover called Opportunity shows the location of crystalline hematite. This is a case of backward presence. It makes the hematite present in our space, through a stratification of communication and spatial layers. On the other hand, (not we but) the scientists who control Opportunity are also forward present on Mars, as interactive agents.

Telepistemic technologies have evolved dramatically since Galileo discovered the four satellites of Jupiter with his own telescope. Nevertheless, by looking at pictures on the screen of a computer we (you and I, not the NASA scientists mentioned above) are no more present on Mars now than he was on those satellites.

Mere word playing, one may reply. Forward and backward presence is just like active and passive sentences: there is no substantial difference between "Peter drives the car" and "the car is driven by Peter". You are where I am, I am where you are. The distinction is linguistically possible but conceptually useless.

The impatient complaint is understandable but unjustified. These are different cases, they should not be confused and the distinction between forward and backward presence is no mere hair-splitting. First of all, it helps to clarify that "local" and "remote" are indexical concepts. Like other words and concepts, such as "I”, "now", "here”, "my” 
and so forth, they acquire a different meaning depending on the position of the observer. The observer's LSO is not the observed's LSO, obviously. It follows that SO is correct in stressing the need for an explicit statement of where the observer is before talking of telepresence. There is no obviously privileged space to be defined as local. Call this an anti-localist thesis.

Second, presence at its best is usually full, that is, both forward and backward presence. The entity is present in RSO as an interactive agent (full forward presence) and it also observes herself locally as being remotely present (backward presence). It is this feedback function that allows the agent to control her interactions with the remote environment.

Third, but not less importantly, being able to understand the difference between the two types of presence means equipping ourselves with a powerful conceptual tool that can help us to frame some ethical problems far more accurately. Some distinctions can be subtle, but this usually means that they are sharper and cut better. We have seen that interactive pornography is a case of what we can now call forward presence. Let us have a look to informational privacy as a case of backward presence.

\section{Privacy: from Intrusion to Abduction}

The literature in computer ethics on privacy is vast and constantly growing (for a review and further references see chapter 5 in Tavani [2003]), so it is not my intention here even to sketch the current debate. The problem is well known in its outlines. What I wish to point out is the need to acquire the correct overall perspective when approaching it.

Privacy is often discussed topologically, i.e., in terms of spaces. But depending on how one understands presence, two fundamental scenarios become available.

If telepistemics is defined in terms of remote presence of the observer - what has been called above as forward presence - it is natural to slip into a view that equates the observed's privacy to the protection of her (local or remote) space, whether physical, psychological or informational. One may then be further inclined to apply familiar concepts of space ownership: a right to privacy may be an exclusive right to own and use one's own information or information about oneself, for example. And since information does not need to be removed to be stolen - as it happens with a car, for example - this 
further reinforces the view that privacy is ownership of an informational space, which is not subtracted to the owner when exploited by someone else. The result is a metaphorical conceptualization of privacy breach as trespassing. Illegal or simply unauthorised access to a web site or a database, or common cases of digital surveillance are portrayed as intrusions in someone else's space or place. It is indicative that the standard line of defence by the intruder - "the gate was open" or "there was no gate" - is not rejected as irrelevant, but rather as pertinent yet mistaken, with a "yes, but" kind of rebuttal.

The problem with this approach is that privacy is often exercised in public spaces, that is, in spaces which are not only socially and physically public - a street, a car park, a pub - but also informationally public - anyone can see the newspaper one buys, the bus one takes, the t-shirt one wears, the drink one is ordering. The tension is obvious. There are about 1.5m cameras monitoring public places in Britain. According to one estimate, the average Briton is recorded by CCTV cameras 300 times a day (The Economist [Jan 23rd 2003]. How could the telepresence of an observer through a CCTV system operated by a bank on a street, for example, be a breach of the observed's privacy if the observer is accessing a space which is public in all possible senses anyway? Why the shop records of the customer's purchase of a t-shirt may breach the latter's privacy, if what one wears is visible to all? Attempts at solving these apparent inconsistencies result in strange geometries of overlapping spaces and exercises in conceptual contortionism. The problem is at the origin: an analysis of telepistemics and hence privacy as forward presence is simply not very helpful.

Consider now the conclusion reached in the previous section. Once telepistemics is understood as a way of making the observed locally present - what has been defined above as backward presence - a privacy breach is more easily comparable to a case of metaphorical abduction: the observed is moved to an observer's local space (a space which is remote for the observed, recall that $\mathrm{LSO} \neq \mathrm{RSO}$ ), unwillingly and possibly unknowingly. Of course, what is abducted is only some information; hence no actual removal is in question (recall the example of the car above) bur rather a cloning of the relevant piece of information. And the cloned information is not a space that belongs to the observed and which has been trespassed; it is rather part of the observed herself, or better something that (at least partly) constitutes the observed for what she is. From this 
perspective, privacy becomes a defence of personal identity and uniqueness. The inconsistency concerning private vs. public spaces does not arise any longer: the observed wishes to preserve her integrity as an informational entity even when she is in an entirely public place. After all, kidnapping is a crime independently of where it is committed, whether in public or not. What one buys, wears or does in public belongs to a sphere that is no one's property in particular, but monitoring and recording it subtracts from this public sphere a portion of the information that constitutes the observed, and makes it part of a space that belongs and is controlled only by the observer, to which the observed herself has no other access, and in a way that may be completely transparent to the observed (the observed is often unaware that part of her information is being abducted).

\section{Conclusion}

When we hear someone loudly speaking on a mobile phone near us, perhaps in the constrained space of a train, we are often annoyed. We do not wish to listen to her business, but cannot help it. Paradoxically, we know that that person is breaching our privacy, yet the old model of presence would not enable us to say why. We could not stop being present and hence regain our privacy by just becoming fully aware of the technologically mediated nature of the experience. And our privacy is certainly not being breached because she is entering in our informational space: we, after all, are the ones who do the listening. It is because she is abducting us into her informational space, forcing us to be telepresent in her space despite our wills. Our privacy is affected because this is a case of imposed backward presence.

The previous is just an example of the kind of approach made possible by the analysis of telepresence developed in this paper, where the old model of presence as epistemic failure has been criticised and an alternative model of presence as successful observability (SO) has been defended as more satisfactory.

The new model SO is based on the general principle that, to be (tele)present is to be the value of a typed variable of a level of abstraction (LoA). SO is no longer Cartesian, for it does not privilege the subjective, internal perception (or lack thereof) of a technologically-mediated displacement. The new model is not even mass-mediatic, for it 
does not refer to social or shared spaces of communication or fiction. SO is a model developed on principles - anti-psychologism, non-Cartesianism, LoA methodology and minimalism in the ontological assumptions - which are at the roots of the philosophy of information (Floridi [2002]). According to SO, what matters in the analysis of presence is the occurrence and flow of information. An entity is forward present in a remote space if it is successfully observed at a LoA in RSO, either as a property-bearer or as a source of change or action/interaction, that is, as some kind of informational object. An entity is backward present in a local space if it is successfully observed at a LoA in LSO at least as a property-bearer, that is, again, as some kind of informational object. Adopting the LoA methodology allows one to specify the ontological commitment in the assessment of presence, while avoiding any (intrinsically unreliable and inevitably opaque) psychologism or qualitative phenomenological description based on the agent's reports of subjective experiences.

We have seen that the SO model provides a new approach to important ethical issues, such as interactive pornography and informational privacy. Whether the new model can withstand criticism and prove to be fruitful is a question open to further research, but one thing remains unchallenged: the more telepresence becomes an ordinary phenomenon, involving an increasing number of people, the more important it will become to understand its nature and its ethical implications in ways that may be utterly unprecedented and unexpected. ICTs increase the ontic and epistemic power of human agents enormously. With these demiurgic capacities are associated equally vast moral issues. More understanding seems the only key to their proper management. After all, the Queen has responsibilities unknown to the Pawn.

\section{Acknowledgements}

I am grateful to Rita Lauria for her kind invitation to contribute to this special issue of Presence and for her several suggestions on the format and contents of this paper. Gianluca Paronitti read a previous draft and provided useful comments. I'm very much in debt to the three anonymous referees of the journal for their valuable suggestions. I remain the only person responsible for any remaining mistakes. 


\section{References}

Biocca, F. 2001, "Inserting the Presence of Mind into a Philosophy of Presence: A Response to Sheridan and Mantovani and Riva", Presence: Teleoperators and Virtual Environments, 10(5), 546-57.

Floridi, L. 2002, "What is the Philosophy of Information?" Metaphilosophy, 33(1-2), 123-45. Preprint http://www.wolfson.ox.ac.uk/ floridi/pdf/wipi.pdf

Floridi, L., and Sanders, J. W. 2004, "The Method of Abstraction" in Yearbook of the Artificial. Nature, Culture and Technology. Models in Contemporary Sciences, edited by M. Negrotti (Bern: Peter Lang). Preprint http://www.wolfson.ox.ac.uk/ floridi/pdf/tmola.pdf

Goldberg, K. (ed.) 2000, The Robot in the Garden: Telerobotics and Telepistemology in the Age of the Internet (Cambridge, Mass.; London: MIT Press).

Ijsselsteijn, W. 2002, "Elements of a Multi-Level Theory of Presence: Phenomenology, Mental Processing and Neural Correlates" in Proceedings of Presence 2002 pp. 245-259. Universidade Fernando Pessoa, Porto, Portugal, Oct. 9-11, 2002.

Ijsselsteijn, W., and Harper, B. 2001, "Virtually There? A Vision on Presence Research" in Presence-Ist 2000-31014 Ec Public Deliverable (D2). Draft Version 1, December. Text available online.

Ijsselsteijn, W. A., de Ridder, H., Freeman, J., and Avons, S. E. forthcoming, "Presence: Concept, Determinants and Measurement", in Proceedings of the SPIE, Human Vision and Electronic Imaging, $V$, 39, 59-76, paper originally presented at Photonics West - Human Vision and Electronic Imaging, San Jose, CA, 23-28 Jan. 2000.

Lauria, R. 2001, "In Answer to a Quasi-Ontological Argument: On Sheridan's "Toward an Eclectic Ontology of Presence" and Mantovani and Riva's "Building a Bridge between Different Scientific Communities"'", Presence: Teleoperators and Virtual Environments, 10(5), 557-63.

Lombard, M., and Ditton, T. 1997, "At the Heart of It All: The Concept of Presence", Journal of Computer-Mediated Communication, 3(2). http://www.ascusc.org/jcmc/vol3/issue2/lombard.html

Mantovani, G., \& Riva, G. 2001, "Building a Bridge between Different Scientific Communities: On Sheridan's Eclectic Ontology of Presence", Presence:

Teleoperators and Virtual Environments, 10(5), 537-43.

Mantovani, G., and Riva, G. 1999, "'Real" Presence: How Different Ontologies Generate Different Criteria for Presence, Telepresence, and Virtual Presence", Presence: Teleoperators and Virtual Environments, 8(5), 540-50.

Minsky, M. 1980, "Telepresence", Omni Magazine, 2(9), 45-51.

Sacau, A., Gouveia, L. B., N., R., Gouveia, F. R., and Biocca, F. 2003, "Presence in Computer-Mediated Environments: A Short Review of the Main Concepts, Theories, and Trends", in Proceedings of IADIS International Conference eSociety 2003, Lisbon, Portugal, 3-6 June 2003. Text available online.

Schuemie, M. J., van der Straaten, P., Krijn, M., and van der Mast, C. A. P. G. 2001, "Research on Presence in Virtual Reality: A Survey", CyberPsychology \& Behavior, 4(2), 183-200. 
Sheridan, T. 1999, "Descartes, Heidegger, Gibson, and God: Toward an Eclectic Ontology of Presence", Presence: Teleoperators and Virtual Environments, 8(5), 551-59.

Tavani, H. T. 2003, Ethics and Technology: Ethical Issues in an Age of Information and Communication Technology (New York: John Wiley \& Sons).

The Economist Jan 23rd 2003, Survey: The Internet Society.

Zahorik, P., and Jenison, R. L. 1998, "Presence as Being-in-the-World", Presence:

Teleoperators and Virtual Environments, 7(1), 78-89. 